\title{
Confinement effect on the dynamics of non-equilibrium concentration fluctuations far from the onset of convection
}

\author{
Cédric Giraudet, ${ }^{1,2}$ Henri Bataller, ${ }^{1}$ Yifei Sun, ${ }^{3}$ Aleksandar \\ Donev, ${ }^{3}$ José M. Ortiz de Zárate, ${ }^{4}$ and Fabrizio Croccolo ${ }^{1,5}$ \\ ${ }^{1}$ Laboratoire des Fluides Complexes et leurs Réservoirs, \\ Université de Pau et des Pays de l'Adour, 64600 Anglet, France \\ ${ }^{2}$ Erlangen Graduate School in Advanced Optical Technologies (SAOT), \\ Friedrich-Alexander-Universität, 91052 Erlangen, Germany \\ ${ }^{3}$ Courant Institute of Mathematical Sciences, New York University, New York, NY 10012, USA \\ ${ }^{4}$ Departamento de Física Aplicada I, Facultad de Física, Universidad Complutense, 28040 Madrid, Spain \\ ${ }^{5}$ Centre National d'Etudes Spatiales (CNES), 2, Place Maurice Quentin, 75001 Paris, France
}

(Dated: October 12, 2016)

\begin{abstract}
In a recent letter [C. Giraudet et al., Europhys. Lett. 111, 60013 (2015)] we reported preliminary data showing evidence of a slowing down of non-equilibrium fluctuations of the concentration in thermodiffusion experiments on a binary mixture of miscible fluids. The reason for this slowing down was attributed to the effect of confinement. Such tentative explanation is here experimentally corroborated by new measurements and theoretically substantiated by studying analytically and numerically the relevant fluctuating hydrodynamics equations. In the new experiments presented here, the magnitude of the temperature gradient is changed, confirming that the system is controlled solely by the solutal Rayleigh number, and that the slowing down is dominated by a combined effect of the driving force of buoyancy, the dissipating force of diffusion and the confinement provided by the vertical extension of the sample cell. Moreover, a compact phenomenological interpolating formula is proposed for easy analysis of experimental results.
\end{abstract}

PACS numbers: 05.40.-a, 05.70.Ln, 47.11.-j, 42.30.Va

\section{INTRODUCTION}

Non-equilibrium thermodynamics (NET) is a basic tool to describe and understand the physics of systems subjected to gradients of intensive properties (in particular, of temperature) at a given time [1]. NET results and descriptions are instrumental in a large range of disciplines like physics of fluids, soft matter physics, astrophysics, statistical physics, biology, metallurgy and many others [1, 2]. NET applied to liquid mixtures shows that, when subjected to a stationary temperature gradient, molecules drift along this gradient due to Soret effect until a stationary concentration gradient is established [3]. This separation is induced by different affinities of fluid molecules to 'heat' [4] and allows a very refined control of concentration profiles in the fluid while giving the ability to investigate intimate properties of fluids like molecular interactions (chemical potentials) [5 7, as well as biomolecular interactions 8 .

A complete description of non-equilibrium systems must include spontaneous non-equilibrium fluctuations (NEFs) whose static and dynamic properties are quite different from those in the equilibrium state, mainly because of their long-ranged nature 9-11] which is not restricted to the proximity of a critical point. Thus, understanding NEFs is a basic question in the physics of non-equilibrium fluids and can lead not only to a deeper insight of transport phenomena like free diffusion or thermodiffusion [12 14, but also of new phenomena like NE fluctuation-induced (Casimir) forces [15 18.

Throughout this paper we shall consider the partic- ular NE system of a thin slab of a binary fluid mixture, of thickness $L$, subjected to a stationary temperature gradient parallel to gravity. This particular NE system has received a lot of attention in the scientific literature, where it is commonly referred to as the binary Rayleigh-Bénard problem [19. Most studies refer to the appearance of convection in this system, and many to the interesting scenario of pattern formation above the convection threshold. However, here, we consider only cases in which the quiescent convection-free state is stable. In particular, we study the concentration fluctuations that spontaneously appear around the stationary profile with uniform concentration gradient $\boldsymbol{\nabla} c$, established by the Soret effect. Here and in the following, $c$ is the mass fraction of the denser component of the fluid mixture. Since the quiescent state is stable, any spontaneous fluctuation will eventually decay and our focus is on the dynamics of this non-equilibrium concentration fluctuations (c-NEFs).

It has been theoretically known for some time 20, 21 that, if confinement effects are neglected, the c-NEFs in this system decay exponentially with a well-defined decay time that depends on the (horizontal, perpendicular to gravity and the applied gradient) wave number $q$ of the fluctuations as:

$$
\left.\tilde{\tau}(\tilde{q})\right|_{d+g}=\frac{\tilde{q}^{2}}{\tilde{q}^{4}-R a_{s}},
$$

where we use dimensionless wave numbers $\tilde{q}=q L$ and decay times $\tilde{\tau}=\tau / \tau_{s}, \tau_{s}=L^{2} / D$, being $D$ the mutual diffusion coefficient of the binary mixture and $\tau_{s}$ the typi- 
cal solutal time it takes diffusion to traverse the thickness of the sample.

Here we are adopting the approximation of large Lewis numbers, that implies vanishing of temperature fluctuations. In this case the number of control parameters reduces to one, as compared to the two control parameters, e.g., the Rayleigh number and the separation ratio, corresponding to full binary Rayleigh-Bénard convection [22]. This is explicitly shown in Eq. (1) through the appearance of the dimensionless solutal Rayleigh number $R a_{s}$, which is the main control parameter of this NE problem [23, 24]:

$$
R a_{s}=-\frac{\beta_{s} \mathbf{g} \cdot \nabla c}{\nu D} L^{4},
$$

where $\beta_{s}=\rho^{-1}(\partial \rho / \partial c)_{T, P}$ is the solutal expansion coefficient, $\rho$ the fluid density, $\mathbf{g}$ the gravity acceleration vector and $\nu$ the kinematic viscosity.

Note that the decay times predicted by Eq. (1), as a function of the wave number, display two quite different behaviors at large and small values, defining two different dynamics. Separating these two regimes one can define a solutal 'characteristic' wave number $q_{s}^{\star}$

$$
\left|R a_{s}\right|=\left(q_{s}^{\star} L\right)^{4},
$$

associated with a 'characteristic' (horizontal) size $\Lambda_{s}^{\star}=$ $2 \pi / q_{s}^{\star}$ of the fluctuations that exhibit different dynamics. Indeed, for large enough wave numbers $q \gg q_{s}^{\star}$ diffusion is the dominant process and c-NEFs time decays are the typical diffusive ones:

$$
\left.\tau(q)\right|_{d}=\frac{1}{D q^{2}} \quad \text { for } \quad q \gg q_{s}^{\star},
$$

On the other hand, for small enough wave numbers $q \ll q_{s}^{\star}$ gravity quenches c-NEFs by means of buoyancy, resulting in a faster decay as:

$$
\left.\tau(q)\right|_{g}=\frac{q^{2} L^{4}}{D\left|R a_{s}\right|} \quad \text { for } \quad q \ll q_{s}^{\star} .
$$

Note that Eq. (5) implies non-diffusive behaviour due to gravity, with fluctuations of larger length scale decaying faster. This unusual behavior, consistent with the approximations adopted to obtain Eq. (1), results rather non-intuitive 25 if extrapolated to $\tilde{q} \rightarrow 0$. Further notice that Eq. (1) is only valid for negative $R a_{s}<0$, while it is known that the quiescent state in this problem is stable for a range of positive $R a_{s}<720$ [26. Confinement effects, neglected when obtaining Eq. (1), must be included in the theory of c-NEFs to overcome these two failings, predicting decay times valid up to the critical $R a_{s}$, and with a sensible limit at $q \rightarrow 0$. Our purpose here is to present such an extension of the theory.

The investigation of spontaneous thermodynamic fluctuations is not only interesting from a purely theoretical point of view. These fluctuations can be observed experimentally by optical techniques sensitive to inhomogeneities in the refractive index $n$ [1] of a well-controlled transparent NE system. Initial experimental studies used dynamic light scattering, which retrieves both decay times and intensities of fluctuations as a function of the scattering wave vector [20, 21]. Due to the difficulty of measuring at very small scattering angles, dynamic light scattering is limited to relatively large wave numbers only. More recently, direct imaging techniques like Shadowgraph and Schlieren have been successfully used to investigate c-NEFs, covering much smaller wave vectors where effects of gravity and confinement become visible [14, 27]. For example, dynamic shadowgraph made it possible to detect the non-diffusive decay of c-NEFs due to gravity [12, 14, 28, predicted by Eq. (1), and the two different associated dynamics of Eqs. (4) and (5). Not only the dynamics of c-NEFs has been investigated when a steady concentration gradient is induced by the Soret effect,

$$
\boldsymbol{\nabla} c=-c(1-c) S_{T} \boldsymbol{\nabla} T
$$

but also for the equivalent case of a pure concentration gradient (isothermal mass diffusion) [25, 29].

Hence, we initiated a research program on confinement effects in c-NEFs comprising both theory and experiments. In a first paper [14] we reported preliminary numerical, theoretical and experimental evidence that, due to the presence of boundaries in the direction of the gradient, not only the intensity 27] but also the dynamics of c-NEFs is modified at very small $q$, exhibiting a dramatic slowing down. Equations (1) and (5) do no longer hold at $q \rightarrow 0$, and a diffusive behavior similar to that of Eq. (4) is recovered, but with a renormalized diffusion coefficient. Such result is here experimentally corroborated by new measurements, in which $R a_{s}$ is tuned by changing the temperature difference $\Delta T$ applied over the sample, and theoretically substantiated by studying analytically and numerically the relevant fluctuating hydrodynamics equations [14, 27.

Experimental investigation of the dynamics of c-NEFs has been proposed as a novel technique for the simultaneous measurement of diffusion and thermal diffusion coefficients in binary mixtures [12, 62, with a possible extension to ternary and multi-component mixtures 63 65. A quick and robust method to obtain reliable values for these coefficients is of high interest to the oil industry, and may open new applications. A good understanding of confinement effects on c-NEFs is mandatory for the practical implementation of these ideas. These possible applications have guided us in the choice of the liquid mixture experimentally investigated, selecting a mixture of a linear and an aromatic hydrocarbon (a crude model of a real oil) with particularly well-known thermophysical properties.

The remainder of the paper is organized as follows: in Section [I] we describe the experimental apparatus and we describe the principle of image analysis we used to extract structure functions. In Section III A and IIIB, we provide details of the Fluctuating Hydrodynamics approach, and of the numerical simulation methods, respectively. The 
experimental results are presented and compared with the theory in Section [IV] Finally in Section $\mathrm{V}$ we summarize our conclusions.

\section{EXPERIMENTAL}

Optical imaging techniques are sensitive to inhomogeneities in the refractive index $n$ of a thermodynamic system, typically as described by the time correlation function $C(q, \tau)$ of refractive index fluctuations at a given wave number $q$. For liquids, the dependence of $n$ on pressure can be neglected, as compared with the dependence on temperature or on composition. Hence, in a binary mixture, $C(q, \tau)$ is related to thermodynamic temperature and concentration fluctuations by [11]:

$$
\begin{aligned}
C(q, \tau)= & \left(\frac{\partial n}{\partial c}\right)^{2} C_{c c}(q, \tau)+\left(\frac{\partial n}{\partial T}\right)^{2} C_{T T}(q, \tau) \\
& +\left(\frac{\partial n}{\partial c}\right)\left(\frac{\partial n}{\partial T}\right) C_{c T}(q, \tau) .
\end{aligned}
$$

where $C_{c c}(q, \tau)$ is the time auto-correlation function of concentration fluctuations, $C_{T T}(q, \tau)$ the time autocorrelation function of temperature fluctuations, and $C_{c T}(q, \tau)$ the time cross-correlation function of concentration and temperature fluctuations. As further discussed below, when the system is subjected to a stationary temperature gradient, each partial time correlation function in Eq. (7) is the sum of an equilibrium (E) and a non-equilibrium (NE) contribution: $C_{i j}(q, \tau)=$ $C_{i j}^{(\mathrm{E})}(q, \tau)+C_{i j}^{(\mathrm{NE})}(q, \tau)[11]$.

The technique of choice for the current investigation is shadowgraphy. For a typical liquid mixture, due to the different intensities and to the range of decay times accesible to our imaging technique, in this work we are only sensitive to c-NEFs, i.e., to $C_{c c}^{(\mathrm{NE})}(q, \tau)$ and over a relatively large range of wave vectors. This principle will guide us during the rest of this investigation.

\section{A. Shadowgraph apparatus and thermodiffusion cells}

Shadowgraphy is a heterodyne near-field scattering technique. This technique is relatively common nowadays, and stems from an intrinsic immunity to stray light which is removed by means of statistical analysis of the images. A schematic representation of our shadowgraph setup is shown in Fig. 1, it consists of a parallel quasi-monochromatic light beam crossing a cell 30 32. and a Charged Coupled Device (CCD) sensor plane to record intensities $I(\mathbf{r}, t)$ integrated along the direction of the gradient. The CCD sensor is placed at $Z_{\mathrm{od}}=$ $(96 \pm 5) \mathrm{mm}$ from the top of the cell (see [12, 14, 33] for more details). Once the parallel beam crosses the cell, no collecting lens is used. Two polarizers (before and after

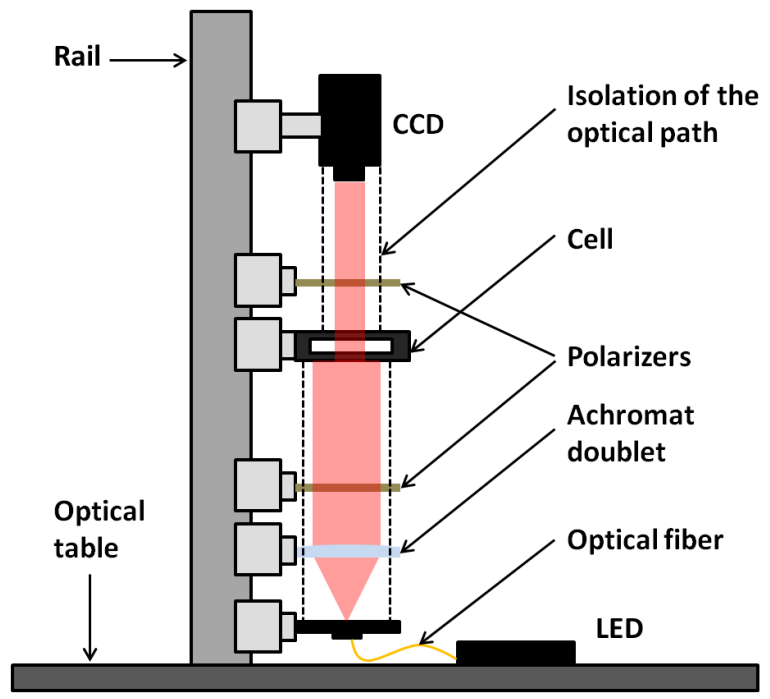

FIG. 1. Scheme of the Shadowgraph apparatus and its optical elements. The entire system is put on an optical table in order to limit stray vibrations.

the measurement cell) are used for beam intensity control, to avoid saturation of the CCD camera while collecting the maximum information from each image. For the measurements presented in this work, two different CCD sensors were used. The first one (IDS, UI-6280SE-M-GL) has a spatial resolution of $2448 \times 2048$ pix $^{2}$ with pixels of $3.45 \times 3.45 \mu \mathrm{m}^{2}$ and a resolution depth of $12 \mathrm{bits} / \mathrm{pix}$; for this sensor images are cropped to a $2048 \times 2048$ pix $^{2}$ resolution, which fixes the minimum wave vector at $q_{\min }=$ $8.89 \mathrm{~cm}^{-1}$. The second sensor (AVT, PIKE-F421B) has a spatial resolution of $2048 \times 2048$ pix $^{2}$ with pixels of $7.4 \times 7.4 \mu \mathrm{m}^{2}$ and a resolution depth of $14 \mathrm{bits} / \mathrm{pix}$; because the size of images is larger than the diameter of optical access to investigate c-NEFs (see thermodiffusion cells description), images from this sensor are cropped to a $1024 \times 1024$ pix $^{2}$ resolution, which fixes the minimum wave vector at $q_{\min }=8.38 \mathrm{~cm}^{-1}$.

The main shape of thermodiffusion cells used is depicted in Fig. 2. two sapphire windows are kept at fixed distance by three precise polystyrene (PS) spacers and sealing o-rings, determining the vertical thickness $L$ of the contained sample fluid. The two sapphire windows are thermally controlled, with a precision of $0.5 \mathrm{mK}$, by two independent Peltier elements having a central hole of diameter $\phi=12.5 \mathrm{~mm}$ to let the light beam to cross them. For window temperature measurement and control, two aluminium plates containing thermistors are located between the sapphires and the Peltier elements. Finally, external to the Peltier elements, there are thick aluminium plates (again with a hole for optical access) that allow passage of water coming from a thermal bath, in order to remove heat excess from the Peltiers, as also shown in Fig. 2 .

To access higher absolute solutal Rayleigh numbers, we also used in this work a thermodiffusion cell specially 


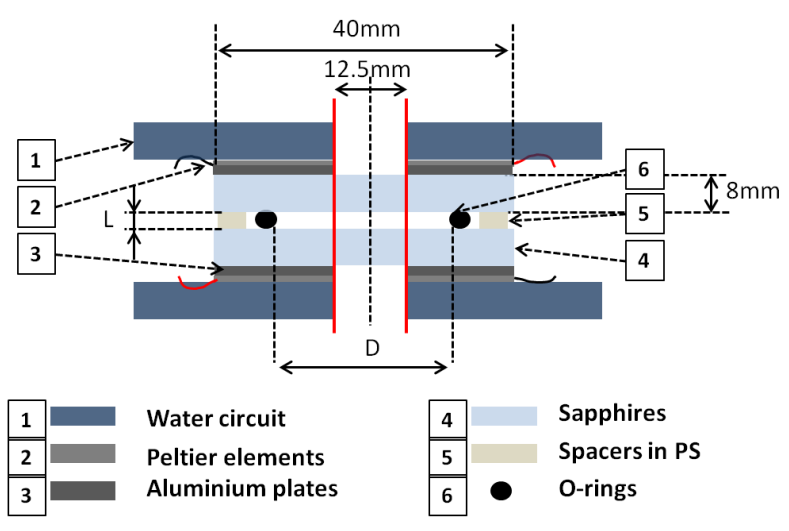

FIG. 2. Scheme of the thermodiffusion cells used in the present studies of c-NEFs.

designed for high pressure measurements, having a thickness $L=5 \mathrm{~mm}$ (see [14, 34 for more information). For the current measurements, this cell was used at atmospheric pressure.

\section{B. Fluid properties}

The analyzed sample is an isomassic $\left(c_{o}=0.5\right)$ binary liquid mixture of tetralin and $n$-dodecane, whose relevant mixture properties are presented in Tab. I. All experiments were performed at atmospheric pressure by applying a stabilizing thermal gradient, with a corresponding $R a_{s}<0$. The separation ratio of the investigated mixture as calculated from the data in Tab. I is positive: $\psi=c_{o}\left(1-c_{o}\right) S_{T} \beta_{s} / \beta_{T}=0.695$, where $\beta_{T}=\rho^{-1}(\partial \rho / \partial T)_{c, P}$ is the thermal expansion coefficient. Hence, negative $R a_{s}$ corresponds to heating from above and under these conditions $\left(R a_{s}<0\right)$, the system is far from the onset of convection: $R a_{s} \ll R a_{s, c}=+720$ [26].

Several negative $R a_{s}$ values were investigated experimentally, as further explained in Sect. IV. The rapid imposition of a temperature difference by heating the fluid mixture from above results in a linear temperature profile across the sample in a thermal time $\tau_{T}=L^{2} / \kappa$, where $\kappa$ is the thermal diffusivity of the fluid. Due to the much smaller value of the mass diffusion coefficient, a nearly linear concentration profile is generated by means of the Soret effect [1, 3] in a much larger solutal diffusion time $\tau_{s}=L^{2} / D$. As further discussed below, and as a consequence of the large value of the Lewis number $(L e=\kappa / D=156$, see Tab. II) of this mixture, temperature and concentration NEFs are temporally uncorrelated and the cross term $C_{c T}(q, \tau)$ in Eq. (7) is equal to zero [22, 27]. Since the investigated mixture has a positive separation ratio, for negative $R a_{s}$ both the temperature and the concentration profile result in a stabilizing density profile [26] and the only variations are due to intrinsic (thermal) fluctuations.
TABLE I. Properties of tetralin- $n$-dodecane mixture at $c=$ $0.5(\mathrm{w} / \mathrm{w})$ and $T=25^{\circ} \mathrm{C}$, from 35] and references therein.

\begin{tabular}{ccc}
\hline \hline Property & Symbol & Value \\
\hline Density & $\rho$ & $0.8407 \mathrm{~g} \cdot \mathrm{cm}^{-3}$ \\
\hline Mass diffusion coefficient & $D$ & $6.21 \times 10^{-6} \mathrm{~cm}^{2} \cdot \mathrm{s}^{-1}$ \\
\hline Thermal diffusivity & $\kappa$ & $9.70 \times 10^{-4} \mathrm{~cm}^{2} \cdot \mathrm{s}^{-1}$ \\
\hline Kinematic viscosity & $\nu$ & $1.78 \times 10^{-2} \mathrm{~cm}^{2} \cdot \mathrm{s}^{-1}$ \\
\hline Soret coefficient & $S_{T}$ & $9.5 \times 10^{-3} \mathrm{~K}^{-1}$ \\
\hline Thermal expansion coefficient & $\beta_{T}$ & $9.23 \times 10^{-4} \mathrm{~K}^{-1}$ \\
\hline Solutal expansion coefficient & $\beta_{s}$ & 0.27 \\
\hline \hline
\end{tabular}

\section{Differential Dynamic Image Analysis}

In our shadowgraphy experiments we acquired sets of 2000 images with a constant delay time of $150 \mathrm{~ms}$. This 'large' acquisition time was essentially set by the frame rate of the CCD camera(s) and actually has the effect of making temperature fluctuations undetectable, because the corresponding decay time is faster in the investigated wave vector range. This means that the term $C_{T T}(q, \tau)$ in Eq. (7) can be set to zero in the analysis of the experimental results.

The intensity images acquired contain a mapping of the sample refractive index fluctuations over space and time averaged along the direction of the gradient. They are typically normalized by their average intensity to get rid of source fluctuations of intensity, $i(\mathbf{r}, t)=$ $I(\mathbf{r}, t) /\langle I(\mathbf{r}, t)\rangle_{\mathbf{r}}$. These intensity patterns are generated at the sensor plane by the heterodyne superposition of the light scattered by the sample refractive-index fluctuations and the much more intense transmitted beam ('local oscillator'). Before extracting modulations of the refractive index, we control normalized images using the procedure described in Refs. [14, 36] to avoid noise in the spectra of c-NEFs due to fluctuations of the acquisition time delay and moving dust in the optical path. In order to retrieve the fluctuating signal, differences of images at given delay times are then calculated as $\delta I(\mathbf{r}, t, \Delta t)=I(\mathbf{r}, t+\Delta t)-I(\mathbf{r}, t)$. The resulting difference images are then $2 \mathrm{D}$-space-Fourier transformed in silico, to separate the contribution of light scattered at different wave vectors. The main steps of the image analysis are illustrated in Fig. 3 .

The dynamics of non-equilibrium concentration fluctuations in a binary liquid mixture can be characterized in terms of the Intermediate Scattering Function (ISF) or, equivalently, normalized time correlation function $f(q, t)$, with $f(q, 0)=1$. Here we extract the ISF by means of the Differential Dynamic Algorithm [12, 25, 29, 37, 38, that is a robust method of getting the so-called structure 


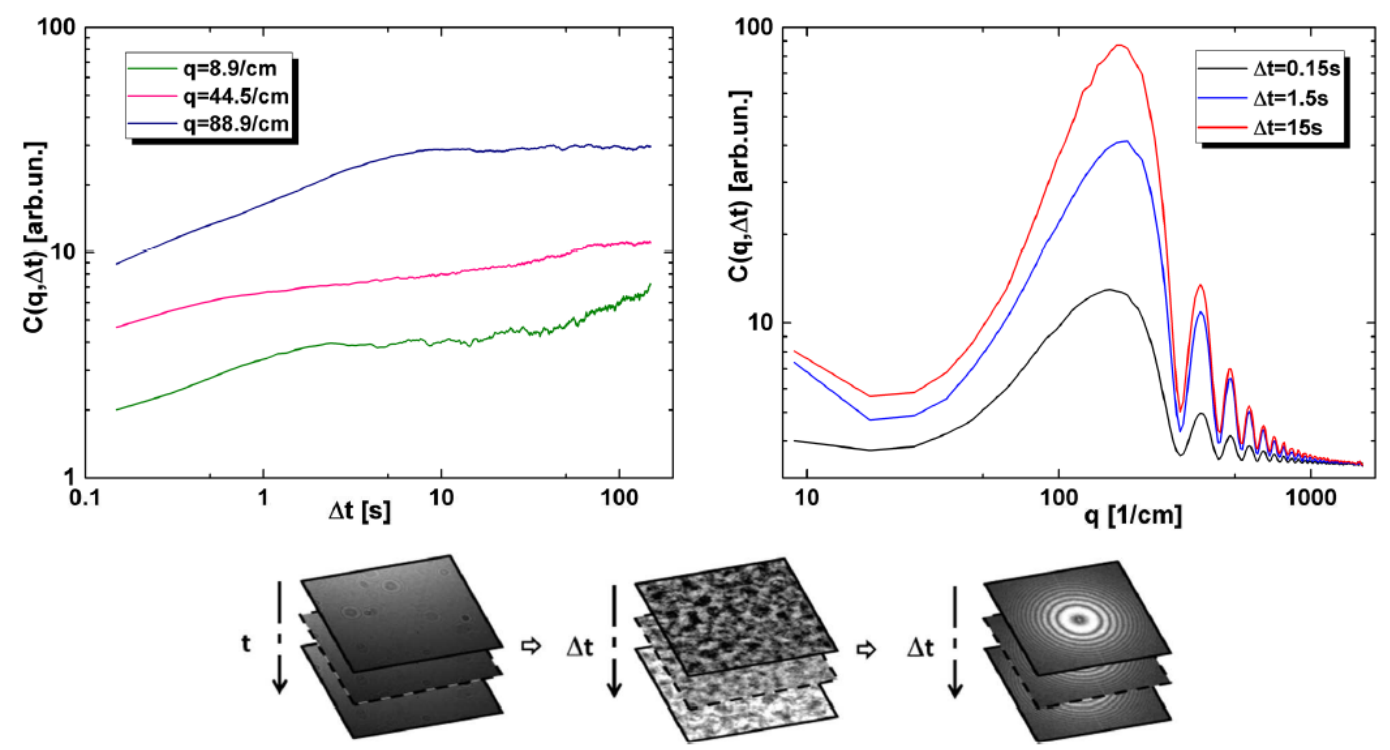

FIG. 3. (top row) Log-log plot of the structure function $C(q, \Delta t)$ as a function of (a) time delay for fixed $q(8.9,44.5$ and 88.9 $\left.\mathrm{cm}^{-1}\right)$ and (b) wave vector for fixed $\Delta t(0.15,1.5$ and $15 \mathrm{~s})$ for $R a_{s}=-2 \cdot 10^{5}$. (bottom row) Stack of normalized images acquired at constant time delay, and the resulting image substractions and their 2D-FFTs.

function:

$$
\begin{aligned}
C(q, \Delta t) & =\left\langle|\Delta i(\mathbf{q}, \Delta t)|^{2}\right\rangle_{t,|\mathbf{q}|=q} \\
& =\left\langle|i(\mathbf{q}, t)-i(\mathbf{q}, t+\Delta t)|^{2}\right\rangle_{t,|\mathbf{q}|=q},
\end{aligned}
$$

with $i(\mathbf{q}, t)=\mathcal{F}[i(\mathbf{x}, t)]$ the 2D-Fourier transform of a normalized image and $\Delta t$ the time delay between the pair of analyzed images, as illustrated in Fig. 3 . The structure function is related to the ISF via [12, 25, 29, 37, 38:

$$
C(q, \Delta t)=2 A\{T(q) S(q)[1-f(q, \Delta t)]+B(q)\},
$$

where $T(q)$ is the optical transfer function of the apparatus [31, 32], $S(q)$ the static structure factor of c-NEFs, $A$ an intensity pre-factor, and $B(q)$ a background including all the phenomena with time-correlation functions decaying faster than the CCD frame rate, such as contributions due to shot noise and temperature fluctuations. Computation of the structure function from a typical dataset of 2,000 images made of 2048x2048 pixels requires parallel processing on a graphical processing unit (GPU) 39] to keep the processing time within the order of one minute.

To obtain $f(q, \Delta t)$ from the experiments, the raw structure function $C(q, \Delta t)$ calculated from the images by Eq. (8), is fit via Eq. (9) once a model for the ISF is adopted. It has been proposed $[12$ that, in first approximation, the ISF can be modeled by a single exponential with decay time $\tau(q)$ depending on the analyzed wave vector $q$. Indeed, for all the wave vectors accessible in the current experiments, we found that the ISF is well modeled by a single exponential over the resolved part of the decay. In this way, for a given $q$, one can fit experimental data points $C(q, \Delta t)$ like the ones shown in Fig. 3 with three adjustable parameters, namely: $A T(q) S(q), \tau(q)$ and $B(q)$. After this preliminary evaluation, an effective ISF is calculated as $f_{\text {eff }}(q, \Delta t)=1-[C(q, \Delta t) / 2 A-B(q)] /[T(q) S(q)]$. For direct comparison with theory and simulations we extract effective decay times from experimental signals as the time needed for $f_{\text {eff }}(q, \Delta t)$ to decay to $1 / e$.

\section{FLUCTUATING HYDRODYNAMICS}

Thermal fluctuations in non-equilibrium systems can be described by fluctuating hydrodynamics (FHD) 11, 40, 61. The full set of FHD equations for a binary fluid mixture in equilibrium were first presented by Foch [41, while for a binary mixture subjected to a stationary temperature gradient by Law and Nieuwoudt [42. In the most general case they are a complicated set of stochastic partial differential equations so that, for practical use, some simplifications adequate for the problem at hand are very convenient. The same series of approximations used in fluid mechanics to simplify the convection problem [22, 43] can be applied in our FHD case, namely: Boussinesq, small Reynolds and large Lewis numbers. Then, one obtains the following stochastic partial differential equations for the fluctuating velocity field $\mathbf{v}(\mathbf{r}, t)$ and for mass concentration $c(\mathbf{r}, t)$ [44],

$$
\begin{aligned}
\rho \partial_{t} \mathbf{v}+\nabla p & =\eta \nabla^{2} \mathbf{v}+\nabla \cdot \Pi-\rho \beta_{s} c \mathbf{g} \\
\boldsymbol{\nabla} \cdot \mathbf{v} & =0 \\
\partial_{t} c+\mathbf{v} \cdot \boldsymbol{\nabla} c & =D \nabla^{2} c,
\end{aligned}
$$

where $\eta=\rho \nu$ is the shear viscosity, $p(\mathbf{r}, t)$ the pressure and $\Pi(\mathbf{r}, t)$ denotes a white-noise stochastic momentum flux due to thermal fluctuations. Nonlinear advective terms in the velocity equation (10a) are neglected in a 
small Reynolds-number approximation. In a Boussinesq approximation, mass balance equation $110 \mathrm{~b}$ reduces to divergence-free velocity fluctuations. Temperature fluctuations are not considered in a large Lewis-number (very fast temperature dynamics) approximation [22, 27]. This approximation is adequate for many liquid mixtures (but not for gases) of positive separation ratio and, in the presence of gravity, for sufficiently large wave numbers $q L>5.18$ [14. Finally, we note that in (10c) we have ignored a stochastic mass diffusion flux, which is responsible for equilibrium fluctuations in the concentration. This is because the non-equilibrium fluctuations induced by the coupling to the velocity equation via the advective term $\mathbf{v} \cdot \nabla c[27$ are much larger than the equilibrium ones.

The stochastic forcing in Eqs. (10) is represented by the random stress $\Pi(\mathbf{r}, t)$. When averaged over fluctuations it vanishes, $\langle\Pi(\mathbf{r}, t)\rangle=0$, while its two-point time correlation function among its components is given by [11, 40, 45]:

$$
\begin{aligned}
\left\langle\Pi_{i j}(\mathbf{r}, t) \Pi_{k l}\left(\mathbf{r}^{\prime}, t^{\prime}\right)\right\rangle=2 k_{\mathrm{B}} T \eta\left(\delta_{i k} \delta_{j l}+\delta_{i l} \delta_{j k}\right) & \\
\times & \delta\left(\mathbf{r}-\mathbf{r}^{\prime}\right) \delta\left(t-t^{\prime}\right),
\end{aligned}
$$

where $k_{\mathrm{B}}$ is Boltzmann's constant. Although Eq. (11) is rigorously demonstrated only for (global) equilibrium systems [11, 40, 46], in the application of FHD to non-equilibrium it is assumed that Eq. (11) continues to be valid, but only after substituting the thermophysical properties in the prefactor by their localequilibrium values [45]. In addition, as previous investigators [45, 47, 48, we shall further assume here that the applied temperature gradient $\nabla T$ is weak and approximate $T \approx T_{o}=298 \mathrm{~K}$ in the prefactor of Eq. (11). Similarly, all other thermophysical properties in Eqs. 101(11) are taken at the average value in the layer for concentration and temperature, as carefully checked to be valid in different experimental conditions [49, 50].

To account for confinement effects, one has to implement realistic boundary conditions:

$$
\mathbf{v}=0 \quad \text { and } \quad \partial_{y} c=\nabla c_{0} \quad \text { at } \quad y=0 \text { and } y=L,
$$

where the concentration gradient induced by the Soret effect $\nabla c_{0}=-c_{0}\left(1-c_{0}\right) S_{T} \nabla T$ ensures that the diffusive mass flux balances the thermodiffusive flux at the impervious walls. Note that here, as well as in Eq. (6), we have used the fact that the gradient is weak and replaced $c(1-c)$ with $c_{0}\left(1-c_{0}\right)$ in the expression (6) of the Soret flux. We note that the Soret effect appears in the FHD model used to interpret the current experiments only through the boundary conditions $(12)$. As already discussed (also in the Experimental Sect. III) the large value of the Lewis number ( $L e=156$ in our case) means that temperature fluctuations decay very fast, so that at the experimental time scales contribute only to the background 22. Neglecting temperature fluctuations causes the Soret effect not to appear in Eqs. (10).
Averaging over fluctuations the FHD equations (10) the term containing the random stress $\Pi(\mathbf{r}, t)$ vanishes and one obtains the deterministic version of the problem. It admits a quiescent solution given by: $\mathbf{v}_{o}=0$, a stationary concentration gradient $\nabla c_{o}$ in the direction of gravity (that we take as $y$, and is parallel to that of the temperature gradient), and the corresponding hydrostatic pressure profile to satisfy $\nabla p_{o}=-\rho \beta_{s} c$ g. Here, we are interested in the spatiotemporal evolution of fluctuations $\mathbf{v}$ and $\delta c$ around such stationary non-equilibrium state. Before presenting our results, we should mention that the linear stability of the problem associated to (the deterministic version of) Eqs. 10 - 12 was studied by Ryskin et al. [26] who showed that $R a_{s}$ is the only control parameter and that the quiescent solution is linearlystable for $R a_{s}<720$.

\section{A. Expansion in approximate hydrodynamic modes}

To solve the FHD equations $100-(12)$ for fluctuations around the non-equilibrium steady state with uniform $\nabla c_{o}$ parallel to gravity ( $y$-direction), we first simplify them keeping only terms linear in the fluctuating fields, then we apply a double curl to the velocity equation (10a) that eliminates pressure. Since the gradient is in the same direction as gravity, perpendicular velocity fluctuations are decoupled from $\delta c$, so that to solve for concentration fluctuations one is left with [11, 27]:

$$
\begin{aligned}
0 & =\nabla^{4} v_{y}-\beta_{s} g\left(\partial_{x}^{2}+\partial_{z}^{2}\right) \delta c+\frac{1}{\rho}\{\nabla \times \nabla \times(\nabla \cdot \Pi)\}_{y}, \\
\partial_{t}(\delta c) & =D \nabla^{2}(\delta c)-\left(\nabla c_{0}\right) v_{y},
\end{aligned}
$$

where $v_{y}$ represents the fluctuations in the fluid velocity component along the direction of the gradient. Notice in the left-hand side of the first of Eqs. (13) that we have further simplified the problem by adopting a large Schmidtnumber approximation [11, 27. This assumption is consistent with the large Lewis-number approach adopted earlier, and it is expected to be a good approximation for most liquid mixtures. For instance, for the tetralindodecane mixture under investigation here, the Schmidt number is $S c=\nu / D=2870$, see Tab. If. We shall return to the validity of this approximation in Sect. IIIB, in connection with the simulations.

The problem of FHD is to solve Eqs. (13) so as to obtain the correlation function of concentration fluctuations (proportional to $f(q, \tau)$ the measured ISF [11, 12]) from the correlation function of the stochastic noise $\Pi(\mathbf{r}, t)$. If one does not consider boundary conditions for the fluctuating fields, Eqs. (13) are readily solved in the Fourier domain [20, 21, 51], leading to a single exponential decay of the ISF with dimensionless decay time given by Eq. (1). As explained in Sect. If this solution without boundary conditions is only meaningful for negative $R a_{s}$. Our goal is to incorporate confinement effects on the dynamics of the fluctuations, what is achieved by adopting boundary 
conditions. The boundary conditions for the fluctuations are:

$$
0=v_{y}=\partial_{y} v_{y}=\partial_{y} \delta c \quad \text { at } \quad y=0 \text { and } y=L,
$$

where the divergence-free condition, Eq. (10b), has been employed. Since confinement (14) is in the direction of the gradient, the problem is most conveniently solved by applying Fourier transforms in time and in the perpendicular $x z$-directions to Eqs. (13), so as to obtain:

$$
\begin{aligned}
0 & =\left(\left[\partial_{y}^{2}-q^{2}\right]^{2} \hat{v}_{y}\right)+\beta_{s} g q^{2} \delta \hat{c}+\hat{F}(\omega, \mathbf{q}), \\
\mathrm{i} \omega \delta \hat{c} & =D\left(\left[\partial_{y}^{2}-q^{2}\right] \delta \hat{c}\right)-\left(\nabla c_{0}\right) \hat{v}_{y},
\end{aligned}
$$

where $q^{2}=q_{x}^{2}+q_{z}^{2}$ is the wave number of the fluctuations in the plane perpendicular to the gradient, and $\hat{F}(\omega, \mathbf{q})$ represents the Fourier transform of the combination of derivatives of the random stress appearing in Eqs. (13).

In a previous publication of purely theoretical nature [27, it was shown that conditions (14) can be implemented by expanding the solution, $\delta \hat{c}(\omega, \mathbf{q}, y)$ of Eqs. 115. in a series of hydrodynamic modes that solve an associated eigenvalue problem while satisfying appropriate boundary conditions. The hydrodynamic modes (eigenfunctions) have single decay times $\tau_{N}(q)$ that are obtained by numerically solving a transcendent equation. Specifically the inverse decay times $\Gamma_{N}=\tau_{N}^{-1}$ are the (multiple) solutions of [27]:

$$
0=\sum_{i=1}^{3} \frac{3 \lambda_{i}(\Gamma)-2 \Gamma}{\Gamma^{2}-9 \lambda_{i}^{2}(\Gamma)} \frac{\operatorname{coth}\left[q^{2}-\frac{1}{3} \Gamma-\lambda_{i}(\Gamma)\right]}{\sqrt{q^{2}-\frac{1}{3} \Gamma-\lambda_{i}(\Gamma)}}
$$

where $\lambda_{i}(\Gamma)$ are the three (complex) cubic roots of the polynomial:

$$
\lambda^{3}-\frac{1}{3} \Gamma^{2} \lambda-\frac{2}{27} \Gamma^{3}-R a_{s} q^{2}=0 .
$$

As a consequence of the oscillatory character of the function coth of complex argument, Eqs. (16)-(17) have an infinite enumerable set of solutions $\Gamma_{N}$ (or $\tau_{N}$ ) for each combination of values: $\left\{q, R a_{s}\right\}$.

The focus of our previous theoretical investigations 27 , 52 was on the static structure factor $S(q)$ of c-NEFs for which, in the particular case of absence of gravity $R a_{s}=0$, a compact analytical expression can be obtained [52]. The dynamics of c-NEFs, although implicitly included [27, 52, was not discussed in detail for arbitrary $R a_{s}$. We proceed next with such an investigation. First of all, it has to be noted that the experimentally observed light intensity is proportional to the intensity of the fluctuations in the concentration averaged along the gradient [11, 27, 31,

$$
\delta c_{\perp}(\mathbf{x}, t)=\frac{1}{L} \int_{0}^{L} \delta c(\mathbf{x}, y, t) d y,
$$

where here $\mathbf{x}$ is a $(2 \mathrm{D})$ position vector in the plane perpendicular to the gradient. Indeed, the shadowgraph theory shows that the spatial spectrum of the timecorrelation function of light fluctuations, once corrected for the optical transfer function of the equipment, i.e., the quantity $I(q, \Delta t)=S(q) f(q, \Delta t)$ in Eq. (9), is given by the correlation function [12, 25, 29, 31, 38:

$$
I(q, \Delta t)=S(q) f(q, \Delta t)=\left\langle\delta \hat{c}_{\perp}(q, \Delta t) \delta \hat{c}_{\perp}^{\star}(q, 0)\right\rangle
$$

of concentration fluctuations Fourier transformed in the plane perpendicular to gravity and the gradient. Notice that, for fluctuations around the quiescent state, the problem has rotational symmetry in that plane, so that $I(q, t)$ depends only on the magnitude of the wave vector q. For the current investigation, following the method of Ref. 27] we have found that, accounting for confinement effects, the ISF $f(q, \Delta t)$ can be theoretically expressed as a series of exponentials:

$$
f(q, \Delta t)=\sum_{N=1}^{\infty} A_{N}(q) \exp \left[-\frac{\Delta t}{\tau_{N}(q)}\right],
$$

The decay times in Eq. 20 are the inverse of the eigenvalues $\Gamma_{N}(q)=1 / \tau_{N}(q)$ from solving Eqs. (16)-(17). The amplitudes $A_{N}$ are analytically related to $\Gamma_{N}$ and $q$. The corresponding expression is too long and complicated to present it here, although we have used it in the numerical discussion that follows. In general, a discussion of the theoretical $f(q, \Delta t)$ of Eq. 20 requires a computation of the decay rates which can be performed numerically only. However, in the limit $q \rightarrow 0$, a full analytical investigation is possible by means of power expansions in $q$, that identifies a clear hierarchy of well-separated $\Gamma_{N}$ 27. In that limit, the slowest mode, i.e., the first term in Eq. (20) dominates, and $f(q \rightarrow 0, t)$ becomes single-exponential in practice. Indeed, it can be shown that the amplitude $A_{1}$ of the slowest mode admits the expansion

$$
A_{1}(q) \stackrel{q \rightarrow 0}{\longrightarrow} 1+\mathcal{O}\left(q^{4}\right)
$$

at small wave numbers; whereas the amplitudes of all other modes, $A_{N}(q)$ with $N>1$, decay to zero proportionally to $q^{4}$ in the same limit. Hence, one concludes that for small $q$ the ISF becomes a single exponential with decay time due to confinement (subscript c):

$$
\left.\tilde{\tau}(\tilde{q} \rightarrow 0)\right|_{c}=\frac{1}{\tilde{q}^{2}\left(1-\frac{R a_{s}}{R a_{s, c}}\right)}=\frac{1}{\tilde{q}^{2}\left(1-\frac{R a_{s}}{720}\right)},
$$

that identifies with $\tau_{1}$ in that limit. Thus, one important conclusion is the prediction of a crossover from Eq. (1) (not-including confinement) at large and intermediate $q$, to the confinement behaviour of Eq. 22 at small $q$. One can estimate the wave number $\tilde{q}_{\mathrm{b}}$ characterizing the crossover between these two behaviors by equating Eqs. (1) and (22). This gives $\tilde{q}_{\mathrm{b}}=\sqrt[4]{R a_{s, c}} \cong 5.2$ independent of $R a_{s}$ and any fluid properties.

Equation (22) gives positive decay times for any $R a_{s}<$ $R a_{s, c}=720$. Although all the data discussed in what follows are for negative $R a_{s}$, we note that the solution (20) 
TABLE II. Experimental conditions for data presented in Figs. 4. 5. Cell height, corresponding solutal Rayleigh number, grid resolutions of the numerical simulations and constant $\alpha$ of Eq. 24. Values of $R a_{s}$ were evaluated from Eqs. (2) and (6) using the thermophysical properties of Table I at average concentration and temperature.

\begin{tabular}{cccc}
\hline \hline \multicolumn{4}{c}{ Temperature difference, $\Delta T=20 \mathrm{~K}$} \\
\hline \hline Cell height, $L$ & $R a_{s}$ & Grid resolution & $\alpha$ \\
\hline $0.7 \mathrm{~mm}$ & $-4 \cdot 10^{4}$ & $280 \times 32$ & 30 \\
\hline $1.3 \mathrm{~mm}$ & $-2 \cdot 10^{5}$ & $300 \times 64$ & 68 \\
\hline $5 \mathrm{~mm}$ & $-1 \cdot 10^{7}$ & $156 \times 128$ & 73 \\
\hline \hline
\end{tabular}

to the FHD equations that incorporates the boundary conditions (12) is meaningful (i.e., it is a decaying function of $\Delta t$ ) for any $R a_{s}<720$, including the range of positive solutal Rayleigh numbers below the convection threshold of the deterministic version of this problem [26, 27.

To complete the theoretical investigation, we numerically evaluated the decay times $\tau_{N}(q)$ and the corresponding amplitudes $A_{N}(q)$ for a range of previously unexplored large and negative values of the solutal Rayleigh number, relevant to the experiments with variable cell height. Previous numerical work 27] considered only small (in magnitude) negative solutal Rayleigh numbers. Here we investigate the $R a_{s}$ values for realistic liquid mixtures that are reported in Table II and find different, much richer, $\Gamma_{N}(q)$ and $A_{N}(q)$ landscapes. The results of these calculations for the three different $R a_{s}$ of Table II are summarized in Fig. 4 as a function of $\tilde{q}$. Each sub-figure corresponds to a different $R a_{s}$, as indicated. For clarity, only the decay times $\tau_{1}(q)$ and $\tau_{2}(q)$ of the two slowest modes, obtained from solving numerically Eqs. (16)-(17), are shown as black and red symbols respectively. Curves representing the theoretical Eqs. (1) and (22) have been also added. One observes how all modes merge in the common Eq. (1) for large $q$, while $\tau_{1}$ is well approximated by Eq. 222 at small $q$.

The top panels of each sub-figure in Fig. 4 show the amplitudes $A_{N}$ of the three slowest modes, which are related to the $\tau_{N}(q)$ by a complicated (but analytical) nonlinear relationship. Even though some numerical convergence problems appear when evaluating the amplitudes of the smallest eigenmodes, the overall picture is clear. For small $q$ the amplitude $A_{1}$ dominates according to Eq. (21), although for $R a_{s}=-10^{7}$ the minimum $q$ for which we were able to compute numerically is still far from the asymptotic limit. The other $A_{N}(N>1)$ decay proportionally to $q^{4}$ in this same limit. At intermediate $q$ and depending on $R a_{s}$, the amplitude of $A_{2}$ (and even of $A_{3}$ ) can be larger than $A_{1}$. This means that the ISF is expected to show signs of multi-exponential behavior as a function of $\Delta t$ in this range of $q$. At large $q \rightarrow \infty, A_{1}$ again becomes the larger amplitude although all modes contribute to the asymptotic $\propto q^{-4}$ dependence expected at this range. As a conclusion, the theoretical ISFs computed from the data in Fig. 4 and Eq. (20), depending on the wave number $q$, exhibit a clear multi-exponential behavior. To compare theoretical ISFs to experiments, we defined an effective decay time $\tau_{\text {eff }}(q)$ that can be extracted by evaluating the time the ISF takes to decay to $1 / e$. These theoretical decay times are also displayed in Fig. 4 as open blue symbols, and will be used later for comparison of theory, experiments and simulations. It can be observed how effective decay times reach the theoretical limit of Eq. 222) at small wave number.

\section{B. Numerical simulations}

We also performed computer simulations of the experimental conditions using finite-volume methods for fluctuating hydrodynamics described in more detail elsewhere [53 55]; here we summarize some key points. The numerical methods have been implemented in the IBAMR software framework 56. The numerical codes solve the equations 10a)-10c expanded to leading order in the magnitude of the fluctuations $\delta c=c-\langle c\rangle$ and $\delta \mathbf{v}=\mathbf{v}-\langle\mathbf{v}\rangle$ around the quiescent $(\langle\mathbf{v}\rangle=0)$ steady state solution of the deterministic equations, as explained in detail in Ref. 55.

Our numerical method solves the complete hydrodynamic equations (10a)-10c in two dimensions with the concentration gradient along the $y$ axis; for this problem there is no difference between two and threedimensional simulations due to the symmetries of the problem. Namely, as already explained, only $q^{2}=q_{x}^{2}+q_{z}^{2}$ matters rather than $q_{x}$ and $q_{y}$ separately, and one can therefore set $q_{z}=0$ without loss of generality.

To simplify the full FHD equations and obtain a relatively simple solution, in Section III A a large Schmidtnumber $(S c=\nu / D \gg 1)$ approximation was adopted, and the limit of equations (10a)- $(10 \mathrm{c})$ as $S c \rightarrow \infty$ was taken. In the linearized setting this over-damped limit amounts to deleting the inertial term $\rho \partial_{t} \mathbf{v}$ in the velocity equation 10a), 22, 26, 27, 55. With this simplification, and after taking a double curl of the velocity equation, one obtains the first of equations (13), with fluid inertia at the left-hand side substituted by zero [27. While neglecting inertial effects is a good approximation at most wavenumbers of interest, it is known that, depending on $R a_{s}$, it fails at sufficiently small wavenumbers due to the appearance of inertial propagative modes [20, 57. (closely related to gravity waves) driven by buoyancy, a phenomenon that cannot be explained within the large $S c$ limit.

With a simple modification of the time-integration algorithm used in the numerical method we can perform simulations with or without the $\rho \partial_{t} \mathbf{v}$ term in the velocity equation (10a), allowing us to study the importance of fluid inertia [55]. In the inertia-less limit we have confirmed that numerical simulations reproduce the results 
of the theoretical calculations of the previous section, based on solving (12)-(13) analytically. In the simulations with inertia we have confirmed that, for the range of $R a_{s}$ probed experimentally, propagative modes appear for the largest $R a_{s}=-10^{7}$ but at wavenumbers $q \lesssim 10$ $\mathrm{cm}^{-1}$ not resolved in the experiments, see Fig. 2 in [55]. For the wavenumbers and Rayleigh numbers experimen-
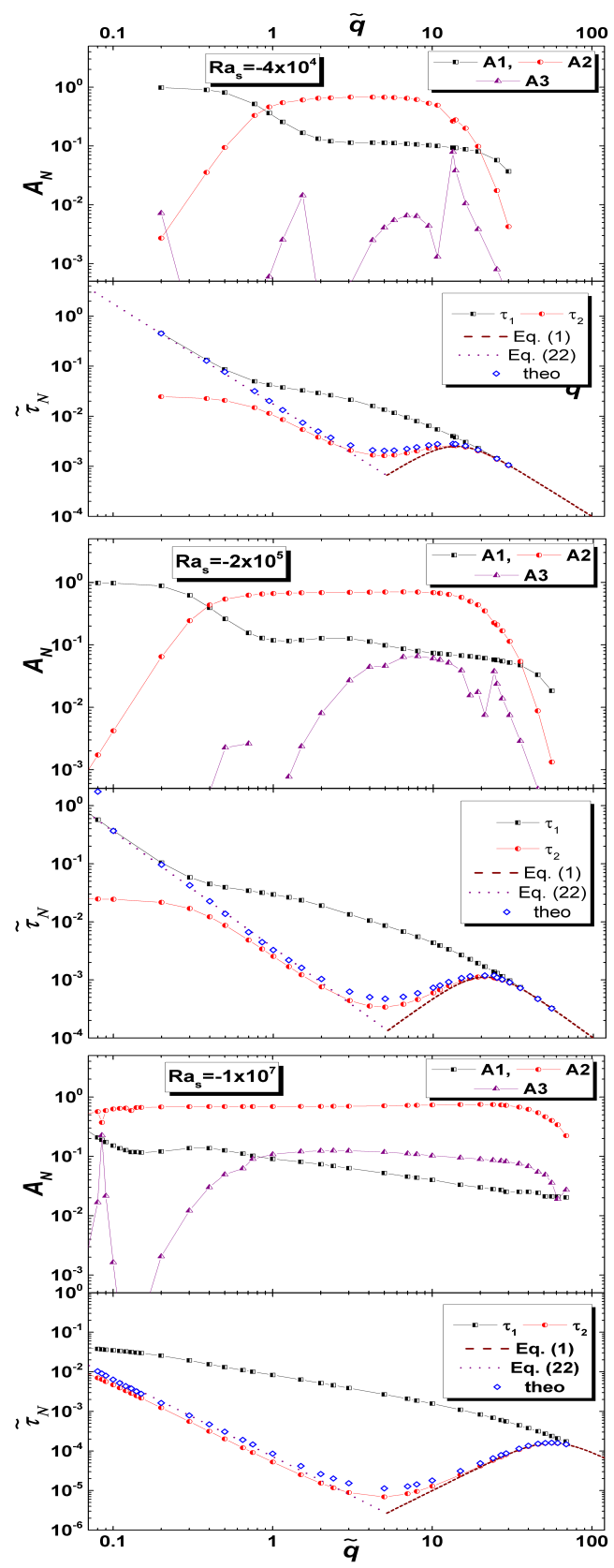

FIG. 4. Summary of theoretical results: Log-log plot of the amplitudes $A_{N}$ (for $N=1,2,3$ ) and of the dimensionless decay times $\tilde{\tau}_{N}=\tau_{N} / \tau_{s}$ (for $N=1,2$ ) as a function of $\tilde{q}$. Top panel is for $R a_{s}=-4 \cdot 10^{4}$, middle panel for $R a_{s}=-2 \cdot 10^{5}$ and bottom panel for $R a_{s}=-1 \cdot 10^{7}$. Eqs. (1), (22) and the theoretical effective decay times, obtained substituting $A_{N}$ and $\tau_{N}$ in Eq. 20 (see text), are also plotted for comparison. tally studied, simulations show negligible effects of inertia on the correlation functions, and the same slow decay at long times is observed for confined fluctuations with or without fluid inertia. Hence, the numerical simulations are used to complement the theory and quantify if fluid inertia is of relevance for the current experimental results.

In our simulations, the domain is periodic along the $x$ direction. At the top and bottom boundaries, $y=0, L$, a no-flux boundary condition (including both Fickian and thermodiffusion (Soret) fluxes) is imposed for the concentration, and a no-slip boundary condition is imposed for velocity, in accordance with Eq. (12). For comparison, we have also performed simulations employing free-slip boundary conditions for the velocity; these show a qualitatively similar behaviour to the results reported here but differ quantitatively indicating the importance of the boundary conditions (confinement).

The physical parameters used in the simulations are the same as reported in Table $\mathbb{I}$ and the temperature difference across the sample is $\Delta T=20 \mathrm{~K}$, heating from the top boundary. The length of the simulation box in the periodic direction (perpendicular to the gradient) is 6.13 $\mathrm{mm}$. The time step size is sufficiently small to resolve the fast viscous dynamics, $\Delta t=5 \times 10^{-3} \mathrm{~s}$. We skip the initial 1250 seconds (in physical time) of the run to allow the steady state to develop, and then collect data for another 6250 seconds. Different sizes of uniform grids were used for the different sample thicknesses, as was summarized in Table 【.

As explained in Sect. IIC, the experimentally observed light intensity, once corrected for the optical transfer function of the equipment, is proportional to the intensity of the fluctuations in the concentration averaged along the gradient [11, 27, 31]. To extract from the simulations the actual ISF to be compared with the experiments, we integrate the concentration along the gravity and gradient coordinate ( $y$-direction) in accordance with Eq. (18) to obtain $\delta c_{\perp}(x, t)$. Since our simulations are $2 \mathrm{D}$, we apply a Fourier transform in the only spatial coordinate left $x$ and, after that, compute the time correlation function of the resulting signal $\delta \hat{c}_{\perp}(q, t)$ in accordance with Eq. 19). After suitable normalization and background subtraction, the resulting time correlation function $I(q, \Delta t)$ is directly related to the experimental ISF, see Eq. (9). For direct comparison with the final result of the experimental analysis, we obtain the effective relaxation time $\tau_{\text {eff }}$ from the simulation results by fitting $I(q, \Delta t)$ to a sum of two exponentials and solving $I\left(q, \tau_{\mathrm{eff}}\right)=I(q, 0) / e$.

\section{EXPERIMENTAL RESULTS AND INTERPOLATING FORMULA}

With the experimental setup and the fluid mixture described in Sect. II two series of experiments were performed. In a first series, the solutal Rayleigh number was varied by changing the separation $L$ between plates and 
keeping constant the temperature difference, $\Delta T=20 \mathrm{~K}$. This procedure allows to cover a relatively large range of $R a_{s}$. Three different spacers were used in the thermodiffusion cell corresponding to three Rayleigh numbers as specified in Table II. This first series was already reported in our recent publication [14] and here we provide further comments on those data.

In a second series of experiments, we keep constant the separation between plates in the thermodiffusion cell at $L=1 \mathrm{~mm}$, but varied the applied temperature difference in the range $4 \leqslant \Delta T \leqslant 20 \mathrm{~K}$. This procedure is simpler experimentally, but only covers the narrower range $-1 \times$ $10^{5} \leqslant R a_{s} \leqslant-2 \times 10^{4}$ of solutal Rayleigh numbers, as indicated in Table III.

For ease of comparison and interpretation, as explained in the previous sections, experimental, theoretical and simulation results for the ISF of c-NEFs were reduced to a single parameter, namely, an effective decay time $\tau_{\text {eff }}$. In our first publication on this topic [14] we reported the experimental $\tau_{\text {eff }}$ corresponding to the first series of experiments, together with the corresponding effective decay times obtained from theory and simulations. A dramatic slowing-down in the dynamics of the fluctuations at $q \rightarrow 0$ was discovered and, by a combination of theory and simulations, attributed to confinement effects.

As it is clear from the details spelled out in Sect. III and not included in our previous publication [14, the theoretical calculation of $\tau_{\text {eff }}(q)$ is only possible numerically, after a long and tedious algebraic process. Hence, from a practical point of view it would be very useful to have an analytical expression for $\tilde{\tau}(\tilde{q})$ for any $R a_{s}$, able to replace Eq. (1) and incorporate the confinement as described by Eq. (22) for small wave numbers. Specifically, one may consider an interpolating formula that crosses over analytically from Eq. 222 at extremely small $\tilde{q}$ to Eq. (1) at larger $\tilde{q}$, and that allows for a swift and physically sensible analysis of experimental results. Next, we shall use the the already published results of the first series of experiments 14 to propose such an interpolating formula, while the second series of new experiments will be employed to check its validity.

Then, in Fig. 5 we report, as filled red symbols in a double-log plot, the experimental $\tau_{\text {eff }}$ obtained from the first series of measurements for $R a_{s}=-2 \cdot 10^{5}$. For completeness, we have also added, as blue and black symbols, the results obtained from theory and simulations, as well as the theoretical predictions of Eq. (1) for $\tilde{q}>\tilde{q}_{\mathrm{b}}$ and of Eq. 22 for $\tilde{q}<\tilde{q_{\mathrm{b}}}$, as dotted curves. Clearly, at small $q$ the experimental data are not compatible with the predictions of Eq. (1) which does not take into account confinement. The deviations observed at small $\tilde{q}$ seem to converge to the theoretical Eq. (22), obtained when the confinement is accounted for. Figure 5 clearly shows that we have observed experimentally the effects of confinement on the dynamics of c-NEFs and the related slowing down for wave vectors smaller than $\tilde{q}<\tilde{q}_{\mathrm{b}}$, as further discussed elsewhere [14].

It is worth mentioning that, for the range of $\tilde{q}$ and the value of $R a_{s}$ displayed in Fig. 5, we found no difference in the $\tau_{\text {eff }}$ obtained from simulations performed with or without the inertial term in the FHD equations. That is the same for the other investigated solutal Rayleigh numbers, not displayed here. This confirms that the possible presence of viscous propagative modes is not an issue in the experimentally accesible parameter range, and that small $q$ deviations from Eq. (1) are indeed due to confinement effects. The theoretical $\tau_{\text {eff }}$ (obtained without fluid inertia) and the simulation $\tau_{\text {eff }}$ (obtained either with or without fluid inertia) agree well in the whole range accessible for these studies, which is a bit larger than the experimentally accessible range. We should also mention that in the experiments no hint of the presence of propagative modes was found, as oscillations in the ISF for instance.

Our next goal is to propose an interpolating analytical formula for $\tau_{\text {eff }}(q)$. We first notice that the only difference between the two theoretical equations is that the $\tilde{q}^{4}$ term of Eq. (1) becomes $R a_{s, c}=720$ in Eq. (22), so we substitute these terms by $R a_{s, c}\left(1+\tilde{q}^{4} / R a_{s, c}\right)$, to arrive at the empirical equation:

$$
\left.\frac{\tau(\tilde{q})}{\tau_{s}}\right|_{d+g+c}=\frac{R a_{s, c}+\tilde{q}^{4}}{\tilde{q}^{2}\left(R a_{s, c}-R a_{s}+\tilde{q}^{4}\right)} .
$$

To evaluate how good is this simple interpolating proposal, we have also displayed in Fig. 5 the raw crossover curve, Eq. (23), for $R a_{s}=-2 \cdot 10^{5}$ as a dash-dotted curve. Clearly, a discrepancy is present between the empirical relation (23) and the data points for wave vectors around $\tilde{q}_{b}=5.2$. The gap can be filled-in by slightly modifying

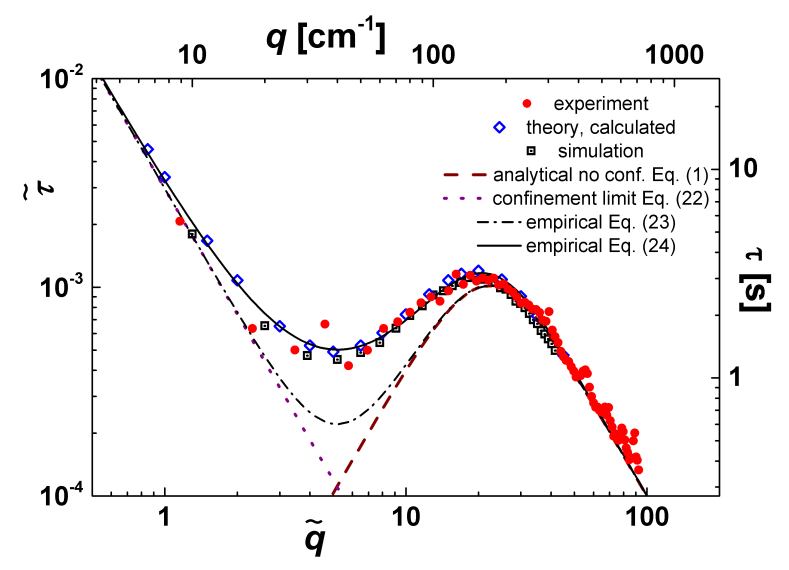

FIG. 5. Log-log plot of the experimental decay times as a function of wave vector for $R a_{s}=-2 \cdot 10^{5}$. Filled red circles are experimental data, open blue diamonds for calculations based on the FHD model, and open-dotted black squares from numerical simulations. The brown dashed line depicts the analytical solution provided by Eq. (1), taking into account gravity and diffusion only; the purple dotted line stands for the confinement limit of Eq. 222. The dashed-dotted black line represents the empirical formula expressed in Eq. 23, while the continuous black line is for Eq. 24 with $\alpha=68$. 
TABLE III. Experimental conditions for the second series of experiments reported in Fig. 6 Temperature difference, solutal Rayleigh number and constant $\alpha$ of Eq. 24. Values of $R a_{s}$ were evaluated from Eqs. (2) and (6) using the thermophysical properties of Table I at averages concentration and temperature.

\begin{tabular}{ccc}
\hline \multicolumn{3}{c}{ Cell height, $L=1 \mathrm{~mm}$} \\
\hline \hline Temperature difference, $\Delta T$ & $R a_{s}$ & $\alpha$ \\
\hline $4 \mathrm{~K}$ & $-2 \cdot 10^{4}$ & 22 \\
\hline $8 \mathrm{~K}$ & $-5 \cdot 10^{4}$ & 30 \\
\hline $12 \mathrm{~K}$ & $-7 \cdot 10^{4}$ & 40 \\
\hline $16 \mathrm{~K}$ & $-9 \cdot 10^{4}$ & 45 \\
\hline $20 \mathrm{~K}$ & $-1 \cdot 10^{5}$ & 50 \\
\hline \hline
\end{tabular}

Eq. 23, adding a term of order $\tilde{q}^{2}$ in the numerator:

$$
\left.\frac{\tau(\tilde{q})}{\tau_{s}}\right|_{d+g+c}=\frac{R a_{s, c}+\alpha \tilde{q}^{2}+\tilde{q}^{4}}{\tilde{q}^{2}\left(R a_{s, c}-R a_{s}+\tilde{q}^{4}\right)},
$$

which does not modify the asymptotic behaviours at large and small $\tilde{q}$. We find that Eq. (24) can be used in practice to fit the experimental data points with the ad hoc dimensionless constant $\alpha$ as an adjustable parameter depending on the Rayleigh number. This is also clearly observed in Fig. 5, where a solid curve represents Eq. (24) for $R a_{s}=-2 \cdot 10^{5}$ and a fitted $\alpha=68$. The need for this fill-in procedure can be justified by noting that the data points are actually effective decay times, obtained by taking the time needed to the correlation function to relax to its $1 / e$ initial value, and thus do not have a clear physical interpretation, as do the decay times of the individual eigenmodes. The values of $\alpha$ obtained by fitting to Eq. (24) the experimental effective decay times of the first series of experiments were reported in Table II.

As mentioned above, a second series of experiments was performed on the same sample of tetralin and ndodecane at averages $c_{0}=0.5$ and $T_{0}=25^{\circ} \mathrm{C}$, but now at fixed thickness $L=1 \mathrm{~mm}$ and tuning the solutal Rayleigh number by varying the temperature difference between plates in the range $\Delta T=[4: 4: 20] \mathrm{K}$. Corresponding values of the solutal Rayleigh number are indicated in Table III In Fig. 6 we report, as open symbols, the experimental effective decay times obtained in this second series of experiments. Temperature difference increases from the top to the bottom of the graph in steps of $4 \mathrm{~K}$, as indicated. Continuous lines represent the decay times predicted by Eq. (24) using the corresponding experimental $R a_{s}$ and $\alpha$ values obtained by fitting to the experimental data points. The $\alpha$ values obtained by this fitting procedure are also reported in Table III for this second series of experiments. Considering data displayed in both Tables III and III we conclude that, in the investigated range, the fitted $\alpha$ values increase while the modulus of $R a_{s}$ increases. In any case, very good agree-

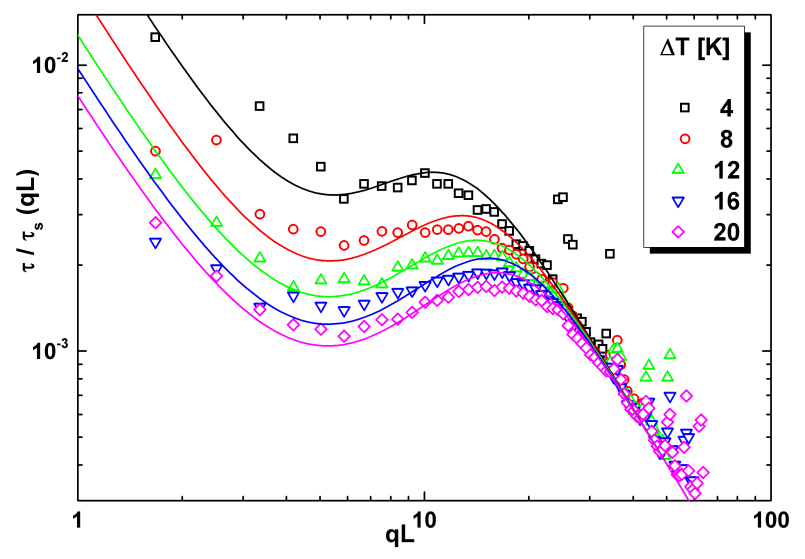

FIG. 6. Log-log plot of the experimental decay times as a function of wave vector for different temperature differences spanning $R a_{s}=-1 \cdot 10^{5}$ to $-2 \cdot 10^{4}$. Open symbols are experimental data, while lines are from Eq. (24), taking into account gravity, diffusion and confinement.

ment is obtained between the experimental results and the proposed interpolating formula. We just note that the experimental data for the lower value of the temperature gradient present a weak signal-to-noise ratio, hence they are somewhat scattered for larger wave vectors.

As already anticipated, we note that for the second set of experiments the range of investigated values of $R a_{s}$ is narrower than for the first set, the reason being the stronger dependency of $R a_{s}$ on $L$ (to a power three). By 'playing' on the confinement $L$ it is possible to 'scan' a larger range of $R a_{s}$ than by merely changing the temperature difference (about 3 orders of magnitude against one order in our case). In any case, the new experimental data measured for the present work and displayed in Fig. 6 strengthen the conclusion 14 that confinement causes an important slowing down of the dynamics of c-NEFs at small wave numbers and that the solutal Rayleigh number is the control parameter of the investigated system.

\section{DISCUSSION AND CONCLUDING REMARKS}

We have investigated experimentally, theoretically and numerically the effective decay time of c-NEFs as a function of the wave number $\tau_{\text {eff }}(q)$. Either in Fig. 5 or in Fig. 6 we observe a similar behavior: As discussed in detail elsewhere [14] for wave numbers smaller than $\tilde{q}_{\mathrm{b}}^{\star} \simeq 5.2$ confinement effects dominate the dynamics of c-NEFs and lead to a diffusive behavior $\left(\tilde{\tau}_{\text {eff }} \propto \tilde{q}^{-2}\right)$ but with a 'confined' diffusion coefficient given by Eq. 222 that can be substantially larger than the mutual diffusion coefficient $D$ of the binary mixture in the bulk phase.

The focus of the present paper has been on intermediate wave numbers, in the range $\tilde{q}_{\mathrm{b}}^{\star}<\tilde{q}<\tilde{q}_{s}^{\star}$, where effective decay times are affected by buoyancy forces. The 
effect of buoyancy on the dynamics depends on the solutal Rayleigh number. In the case of very small $R a_{s}$ buoyancy causes an acceleration in the dynamics of $\mathrm{c}$ NEFs, as evidenced by the presence in $\tau_{\text {eff }}(q)$ of a relative minimum close to $\tilde{q}_{\mathrm{b}}^{\star}$ and a relative maximum close to $\tilde{q}_{s}^{\star}$. Between these two extrema, there is a range of $q$ in which fluctuations of larger (lateral) dimension decay faster, in accordance with Eq. (5). However, for larger $R a_{s}$, the relative extrema at $\tilde{q}_{b}^{\star}$ and $\tilde{q}_{s}^{\star}$ do not appear, and the associated accelerated dynamics due to buoyancy is not observable. We estimate as $R a_{s} \lesssim-10^{4}$ the range of solutal Rayleigh numbers at which an acceleration in the dynamics associated to bouyancy is observable. Without gravity, c-NEFs exhibit the usual diffusive scaling with the standard Fickian diffusion coefficient for all wave numbers, as predicted by linearized fluctuating hydrodynamics [52] and observed in the GRADFLEX microgravity experiments 5860 .

Finally, independently of $R a_{s}$, effective decay times follow a diffusive behavior for larger wave numbers, $\tilde{q}>$ $\tilde{q}_{s}^{\star}$, with a diffusion constant equal to the bulk diffusion coefficient, in accordance with Eq. (4).

The quantitative dynamic shadowgraph observation of c-NEFs has been proposed 12 as a method for the measurement of thermophysical properties of fluids. In particular, quantifying the position of the (relative) maximum in $\tau_{\text {eff }}(q)$ and identifying it with the solutal characteristic wave number $\tilde{q}_{s}^{\star}$ of Eq. (3), an experimental value for $R a_{s}$ can be obtained. From this $R a_{s}$, unknown properties of the mixture can be measured from known values of the other properties appearing in the definition (2) of the solutal Rayleigh number. However, the conclusions of the present work show that one has to be cautious with this procedure. As already discussed, due to confinement effects on the dynamics of c-NEFs, the maximum is not observable for $R a_{s} \gtrsim-10^{4}$. In addition, when the maximum is indeed observable, confinement causes its position not to be equal to $\tilde{q}_{s}^{\star}$. We have observed that, due to confinement, the position of the maximum is displaced to wave numbers smaller than $\tilde{q}_{s}^{\star}$, and becomes equal to $\tilde{q}_{s}^{\star}$ only in the limit $R a_{s} \rightarrow-\infty$. We submit that this may be one of the reasons why the solutal characteristic wave number $\tilde{q}_{s}^{\star}$ obtained from the dynamics is systematically smaller than the one obtained from statics, as has been recently reported [28].

A contribution of this work is the proposition of the interpolating formula 24 , with a single fitting parame- ter $\alpha$ to model the whole curves $\tau(q)$, while having the two correct asymptotic limits at both $q \rightarrow 0$ and $q \rightarrow \infty$. We expect that Eq. (24) will help to alleviate the problems mentioned in the previous paragraph for the use of c-NEFs as a tool to measure thermophysical properties of a liquid mixture. For a sample of unknown thermal diffusion coefficient, Eq. 24 can be used with two fitting parameters, so that from a fitted $R a_{s}$ the Soret effect might be quantified. Obviously, for this program to succeed more experimental and theoretical work will be needed, in particular to better understand if there is a correlation between $R a_{s}$ and the 'fill-in' parameter $\alpha$. Also to check whether the range of $R a_{s}$ for which the interpolating formula (24) is valid can be safely extended, maybe including positive values $R a_{s}>0$. Note at this respect that the denominator in Eq. 24 is positive for all $q$ in the whole range $R a_{s}<R a_{s, c}=720$. We plan to continue research on this topic following this line.

This paper contains a detailed explanation of the theory and simulations supporting the conclusion that the slowing-down in the dynamics of c-NEFs observed [14 at small $q$ is a consequence of confinement and, therefore, this slowing-down appears naturally when boundary conditions are incorporated in the calculation of the time correlation functions. Moreover, we presented additional experimental evidence showing that confinement effects on the dynamics of NEFs can be more important than in the statics (intensity) of these fluctuations. Our present conclusions are highly relevant for the development of optical techniques to investigate thermal diffusion in liquid mixtures

\section{ACKNOWLEDGMENTS}

F.C. acknowledges fruitful discussions with Alberto Vailati, Doriano Brogioli and Roberto Cerbino. F.C. also acknowledges financial support from CNES. We acknowledge financial support from ESA through the SCCO program. JOZ acknowledges partial support from the Spanish State Secretary of Research under Grant No. FIS2014-58950-C2-2-P. A.D. was supported in part by the U.S. National Science Foundation under grant DMS1115341 and the Office of Science of the U.S. Department of Energy through Early Career award number DESC0008271.

Correspondence and requests for materials should be addressed to F.C. (fabrizio.croccolo@univ-pau.fr)
[1] S. R. de Groot and P. Mazur, Non-Equilibrium Thermodynamics (North-Holland, Amsterdam, 1962) dover edition, 1984.

[2] S. Kjelstrup and D. Bedeaux, Non-Equilibrium Thermodynamics Of Heterogeneous Systems (World Scientific, Singapore, 2008).

[3] C. Soret, Arch. Sci. Phys. Nat. 3, 48 (1879).
[4] S. Hartmann, G. Wittko, W. Köhler, K. I. Morozov, K. Albers, and G. Sadowski, Phys. Rev. Lett. 109, 065901 (2012).

[5] S. Wiegand, H. Ning, and R. Kita, J. Non-Equilib. Thermodyn. 32, 193 (2007).

[6] G. Galliero and S. Volz, J. Chem. Phys. 128, 064505 (2008). 
[7] P. Artola and B. Rousseau, Mol. Phys. 111, 3394 (2013).

[8] M. Wolff, J. J. Mittag, T. W. Herling, E. D. Genst, C. M. Dobson, T. P. J. Knowles, D. Braun, and A. K. Buell, Scientific Reports 6, 22829 (2016)

[9] T. R. Kirkpatrick, E. G. D. Cohen, and J. R. Dorfman, Phys. Rev. A 26, 995 (1982).

[10] J. R. Dorfman, T. R. Kirkpatrick, and J. V. Sengers, Annu. Rev. Phys. Chem. 45, 213 (1994).

[11] J. M. Ortiz de Zárate and J. V. Sengers, Hydrodynamic Fluctuations in Fluids and Fluid Mixtures (Elsevier, Amsterdam, 2006).

[12] F. Croccolo, H. Bataller, and F. Scheffold, J. Chem. Phys. 137, 234202 (2012)

[13] M. H. Rausch, A. Heller, J. Herbst, T. M. Koller, M. Bahlmann, P. S. Schulz, P. Wasserscheid, and A. P. Fröba, J. Phys. Chem. B 118, 4636 (2014).

[14] C. Giraudet, H. Bataller, Y. Sun, A. Donev, J. M. Ortiz de Zárate, and F. Croccolo, Europhys. Lett. 111, 60013 (2015).

[15] A. Najafi and R. Golestanian, Europhys. Lett. 68, 776 (2004).

[16] T. R. Kirkpatrick, J. M. Ortiz de Zárate, and J. V. Sengers, Phys. Rev. Lett. 110, 235902 (2013).

[17] T. R. Kirkpatrick, J. M. Ortiz de Zárate, and J. V. Sengers, Phys. Rev. Lett. 115, 035901 (2015).

[18] T. R. Kirkpatrick, J. M. Ortiz de Zárate, and J. V. Sengers, Phys. Rev. E 93, 012148 (2016).

[19] M. C. Cross and P. C. Hohenberg, Rev. Mod.. Phys. 65, 851 (1993).

[20] P. N. Segrè, R. Schmitz, and J. V. Sengers, Physica A 195, 31 (1993).

[21] P. N. Segrè and J. V. Sengers, Physica A 198, 46 (1993).

[22] M. G. Velarde and R. S. Schechter, Phys. Fluids 15, 1707 (1972).

[23] A. Vailati and M. Giglio, Nature 390, 262 (1997).

[24] A. Vailati and M. Giglio, Phys. Rev. E 58, 4361 (1998).

[25] F. Croccolo, D. Brogioli, A. Vailati, M. Giglio, and D. S. Cannell, Phys. Rev. E 76, 041112 (2007).

[26] A. Ryskin, H. W. Müller, and H. Pleiner, Phys. Rev. E 67, 046302 (2003).

[27] J. M. Ortiz de Zárate, J. A. Fornés, and J. V. Sengers, Phys. Rev. E 74, 046305 (2006).

[28] F. Croccolo, H. Bataller, and F. Scheffold, Eur. Phys. J. E 37, 105 (2014).

[29] F. Croccolo, D. Brogioli, A. Vailati, M. Giglio, and D. S. Cannell, Appl. Opt. 45, 2166 (2006).

[30] G. S. Settles, Schlieren and Shadowgraph Techniques (Springer, Berlin, 2001).

[31] S. P. Trainoff and D. S. Cannell, Phys. Fluids 14, 1340 (2002).

[32] F. Croccolo and D. Brogioli, App. Opt. 50, 3419 (2011).

[33] F. Croccolo, F. Scheffold, and A. Vailati, Phys. Rev. Lett. 111, 014502 (2013).

[34] C. Giraudet, H. Bataller, and F. Croccolo, Eur. Phys. J. E 37, 107 (2014).

[35] J. K. Platten, M. M. Bou-Ali, P. Costesèque, J. F. Dutrieux, W. Köhler, C. Leppla, S. Wiegand, and G. Wittko, Phil. Mag. 83, 1965 (2003).

[36] C. Giraudet, PhD dissertation, Université de Pau et des Pays de l'Adour (2015).

[37] F. Croccolo, PhD dissertation, Università degli Studi di Milano (2006).

[38] R. Cerbino and V. Trappe, Phys. Rev. Lett. 100, 188102 (2008).
[39] G. Cerchiari, F. Croccolo, F. Cardinaux, and F. Scheffold, Rev. Sci. Instrum. 83, 106101 (2008).

[40] L. D. Landau and E. M. Lifshitz, Fluid Mechanics (Pergamon, London, 1959) 2nd revised English version, 1987.

[41] J. Foch, Phys. Fluids 14, 893 (1971).

[42] B.M. Law and J. C. Nieuwoudt, Phys. Rev. A, 40 (1989), 3880 .

[43] J. K. Platten and J. C. Legros, Convection in Liquids (Springer, Berlin, 1984).

[44] A. Donev, T. G. Fai, and E. Vanden-Eijnden, J. Stat. Mech. (2014), arxiv:1312.1894.

[45] R. Schmitz and E. G. D. Cohen, J. Stat. Phys. 39, 285 (1985).

[46] R. F. Fox and G. E. Uhlenbeck, Phys. Fluids 13, 1893 (1970).

[47] V. M. Zaǐtsev and M. I. Shliomis, Sov. Phys. JETP 32, 866 (1971).

[48] P. C. Hohenberg and J. B. Swift, Phys. Rev. A 46, 4773 (1992).

[49] W. B. Li, PhD thesis, University of Maryland (1996).

[50] P. N. Segré, R. W. Gammon, J. V. Sengers, and B. M. Law, Phys. Rev. A 45, 714 (1992).

[51] J.V. Sengers, J.M. Ortiz de Zárate, in Thermal Nonequilibrium Phenomena in Fluid Mixtures, Lecture Notes in Physics, vol. 584, ed. by W. Köhler, S. Wiegand (Springer, Berlin, 2002), Lecture Notes in Physics, vol. 584, pp. $121-145$

[52] J. M. Ortiz de Zárate, T. R. Kirkpatrick, and J. V. Sengers, Eur. Phys. J. E 38, 99 (2015).

[53] F. Balboa Usabiaga, J. B. Bell, R. Delgado-Buscalioni, A. Donev, T. G. Fai, B. E. Griffith, and C. S. Peskin, SIAM J. Multiscale Model. Simul. 10, 1369 (2012).

[54] S. Delong, B. E. Griffith, E. Vanden-Eijden, and A. Donev, Phys. Rev. E 87, 033302 (2013).

[55] S. Delong, Y. Sun, B. E. Griffith, E. VandenEijnden, and A. Donev, Phys. Rev. E 90, 063312 (2014), software available at https://github.com/ stochasticHydroTools/MixingIBAMR

[56] B. E. Griffith, R. D. Hornung, D. M. McQueen, and C. S. Peskin, J. Comput. Phys. 223, 10 (2007), software available at http: \\ibamr.googlecode.com.

[57] C. J. Takacs, G. Nikolaenko, and D. S. Cannell, Phys. Rev. Lett. 100, 234502 (2008).

[58] A. Vailati, R. Cerbino, S. Mazzoni, C. J. Takacs, D. S. Cannell, and M. Giglio, Nature Communications 2, 290 (2011).

[59] R. Cerbino, Y. Sun, A. Donev, and A. Vailati, Sci. Rep. 5, 14486 (2015)

[60] F. Croccolo, C. Giraudet, H. Bataller, R. Cerbino, and A. Vailati, Microgravity Sci. and Technol., 28, 467 (2016).

[61] F. Croccolo, J.M. Ortiz de Zárate and J.V. Sengers, Eur. Phys. J. E submitted

[62] F. Croccolo, F. Scheffold, H. Bataller, C. R. Mécanique 341, 378 (2013)

[63] J.M. Ortiz de Zárate, C. Giraudet, H. Bataller, F. Croccolo, Eur. Phys. J. E 37, 77 (2014).

[64] H. Bataller, C. Giraudet, F. Croccolo, J.M. Ortiz de Zárate, Microgravity Sci. Technol. XX, XX (2016). doi: 10.1007/s12217-016-9517-6

[65] P. Martinez-Pancorbo, J.M. Ortiz de Zárate, H. Bataller, and F. Croccolo, Eur. Phys. J. E submitted 